Document downloaded from:

http://hdl.handle.net/10251/98264

This paper must be cited as:

Kurlyandskaya, G.; Yakabchuk, H.; Kisker, E.; Bebenin, N.; García Miquel, ÄH.; Vazquez, M.; Vas'kovskiy, V. (2001). Nonlinear magnetoimpedance effect in FeCoNi ferromagnetic tubes. Chinese Physics Letters. 18(9):1268-1270. doi:10.1088/0256-307X/18/9/337

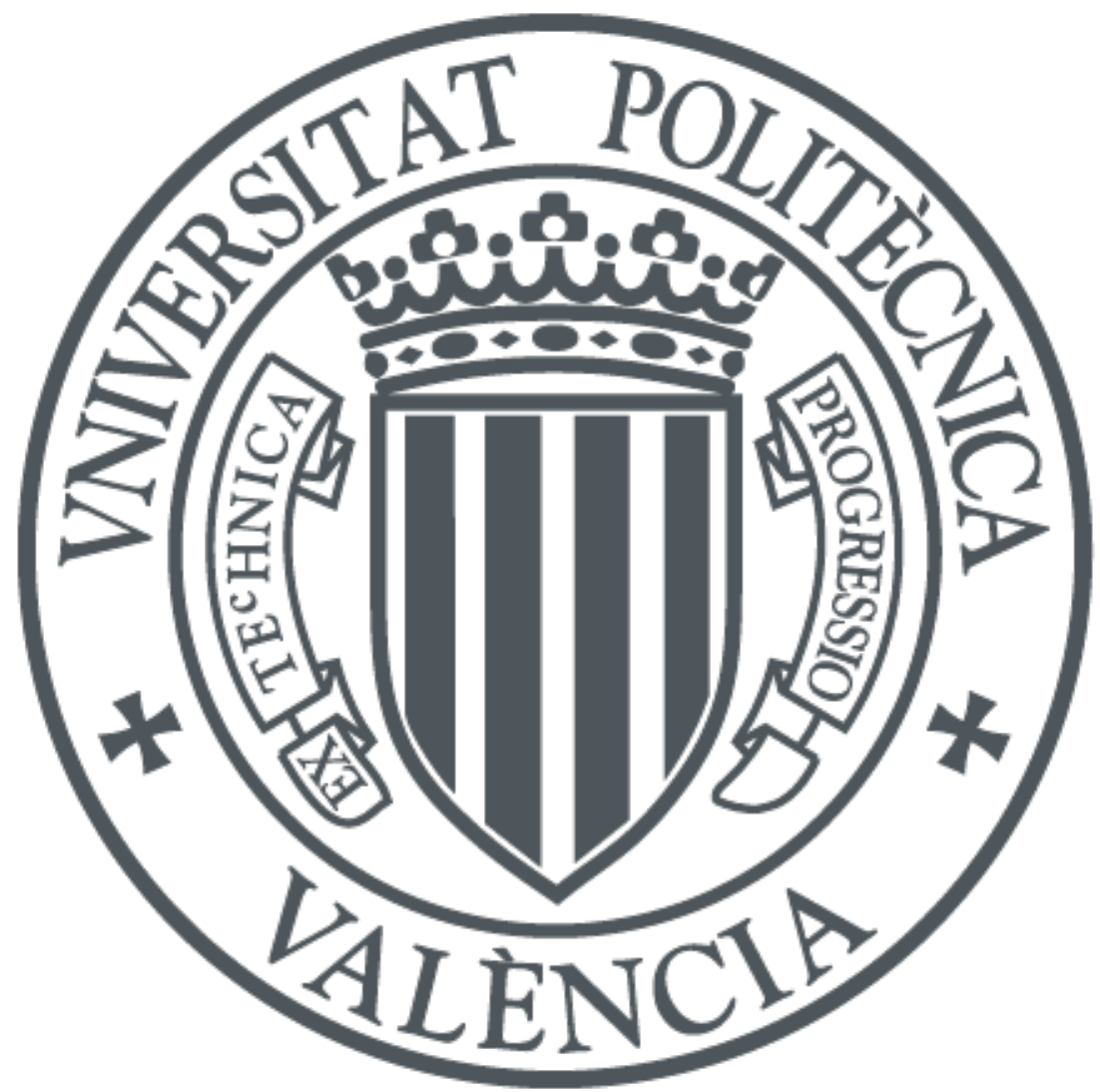

The final publication is available at

https://doi.org/10.1088/0256-307X/18/9/337

Copyright IOP Publishing

Additional Information 


\title{
Nonlinear Magnetoimpedance Effect in FeCoNi Ferromagnetic Tubes
}

\author{
G. V. Kurlyandskaya ${ }^{1,2 *}$, H. Yakabchuk ${ }^{2}$, E. Kisker ${ }^{2}$, N. G. Bebenin ${ }^{3}$, H. García-Miquel ${ }^{4}$, \\ M. Vázquez ${ }^{5}$, V. O. Vas' kovskiy $^{6}$ \\ ${ }^{1}$ Dpto. de Electricidad y Electrica Universidad del Pa Vasco UPV-EHU, Apdo 644, 48080 Bilbao, Spain \\ ${ }^{2}$ Institut für Angewandte Physik, Heinrich Heine Universität Düsseldorf, Universitätstraße 1, \\ Bld.25.33.01.24, D-40225, Düsseldorf, Germany \\ ${ }^{3}$ Institute of Metal Physics UD RAS, Kovalevskaya str. 18, GSP-170, 620219 Ekaterinburg, Russia \\ ${ }^{4}$ Dpto. de Ing. Electrónica, Universidad Politécnica de Valencia, Camino de Vera, s/n, 46022 Valencia, Spain \\ ${ }^{5}$ Instituto de Ciencia de Materiales, CSIC, P.O.Box 155, 28230 Las Rozas (Madrid), Spain \\ ${ }^{6}$ Institute of Physics and Applied Mathematics, Ural State University, Lenin Ave. 51, 620083 Ekaterinburg, Russia
}

\begin{abstract}
The very high (up to $820 \%$ of the magnetoimpedance ratio) and sensitive nonlinear giant magnetoimpedance effect has been studied in the FeCoNi magnetic tubes electroplated onto $\mathrm{Cu}(3 \%) \mathrm{Be}$ nonmagnetic wire for frequencies from 1-10 MHz. Special annealing was carried out in order to induce the magnetic anisotropy. The high harmonic generation was observed and the harmonics show larger variations with the external magnetic field than the fundamental frequency. The super high sensitivity of the harmonics is promising as regards the increase of the sensitivity of magnetoimpedance sensors.
\end{abstract}

The magnetoimpedance (MI) effect is promising for technological applications in micro magnetic field detectors and magnetic recording heads. The phenomenon is classical in nature and for uniform materials results from the penetration depth dependence on the transverse magnetic permeability. The general theory of this effect in a magnetic wire was written by Landau et al..$^{[1]}$ long ago. The discovery by Beach et al..$^{[2]}$ and Panina et al..$^{[3]}$ of the very large magnetoimpedance of $100 \%$ order in amorphous wires has supported the scientific community's interest in the giant magnetoimpedance (GMI). It has been shown experimentally ${ }^{[4-7]}$ and theoretically ${ }^{[8-9]}$ that the GMI value depends on the magnetic properties, in particular magnetic anisotropy because the anisotropy determines the magnetic permeability. The permeability is sensitive to special treatments, so that there are possibilities to modify the GMI responses. Another way to increase the GMI is based on the theoretical $^{[10,11]}$ and experimental ${ }^{[10,12,13]}$ results for multilayered systems with non-uniform conductivity. There are a number of publications on GMI in electroplated $\mathrm{FeNi},{ }^{[12]} \mathrm{FeNiCo},{ }^{[13,14]}$ and $\mathrm{CoP}^{[15]}$ wires. It remains however unclear whether the achieving value of the order of $200-400 \%$ of the GMI ratio is the absolute maximum for those compositions and geometry or whether it is possible to increase the effect. Most of the GMI studies were performed in the classical linear regime. There are, however, publications, ${ }^{[12,16]}$ in which interesting nonlinear effects have been reported.

In this letter, we investigate the extraordinary high and sensitive magnetoimpedance effect and the high harmonic generation in the $\mathrm{FeCoNi}$ magnetic tubes electroplated onto CuBe nonmagnetic wire in order to understand how the nonlinearity modifies the impedance of the wires.

Our sample is $100 \mu$ m diameter nonmagnetic $\mathrm{Cu}(3 \%)$ Be wire electroplated with a $1 \mu \mathrm{m} \mathrm{Fe}{ }_{20} \mathrm{Co}_{6} \mathrm{Ni}_{74}$ layer. The resistivity of the conductive wire was about $3.9 \mu \Omega \cdot \mathrm{cm}$ and that of the magnetic layer about $15 \mu \Omega . \mathrm{cm}$. A current flowing in the wire during the electrodeposition causes the circumferential component of the magnetization due to the presence of the circular magnetic field. The length of the samples was $60 \mathrm{~mm}$. The longitudinal hysteresis loops of the samples were measured by the conventional inductive method at a frequency of $50 \mathrm{~Hz}$. Field annealing was carried out in the Ar protective atmosphere in a magnetic field applied along the axis of the wire for one hour at the temperature of $320^{\circ} \mathrm{C}$. The thermal treatment induced a longitudinal magnetic anisotropy with a magnetization component along the wire axis. The changes of the total impedance were measured through the voltage drop across the sample. The maximum magnetic field was $H_{\max }=$ $\pm 2.6 \mathrm{kA} / \mathrm{m}$. At every magnetic field, the exciting current amplitude was measured and maintained constant regardless of the voltage variations. The magnetoimpedance was measured in the frequency region of $1-10 \mathrm{MHz}$, for ac sinusoidal currents of $I_{\mathrm{rms}}=36$ and $10 \mathrm{~mA}$ in both increasing and decreasing external fields. The magnetoimpedance ratio is defined as $\Delta Z / Z(H)=100\left[Z(H)-Z\left(H_{\max }\right)\right] / Z\left(H_{\max }\right)$ with $H_{\max }= \pm 2.6 \mathrm{kA} / \mathrm{m}$ as the maximum axial field. Term "impedance" belongs to the linear response theory. ${ }^{[1]}$ 

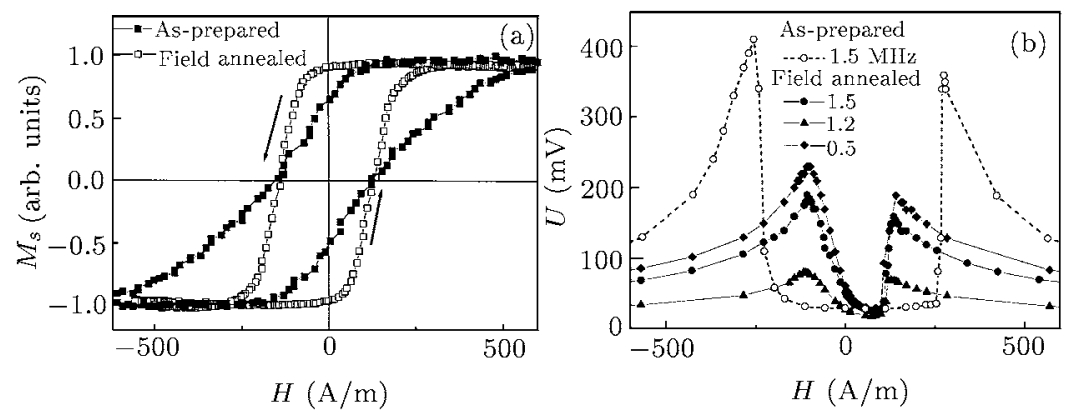

Fig. 1. (a) Longitudinal hysteresis loops and (b) induced voltages with the increasing field at various frequencies of the as-prepared and field annealed FeCoNi electroplated wires.

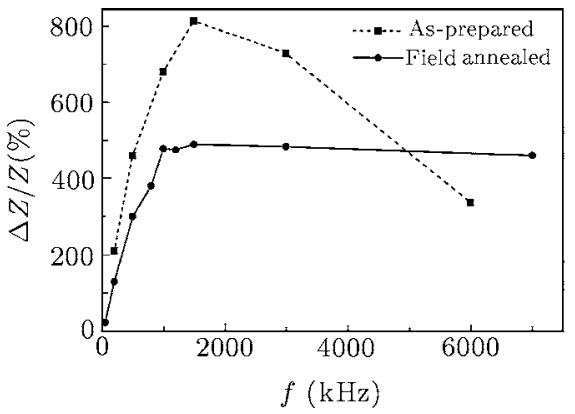

Fig. 2. Frequency dependence of the maximum of the magnetoimpedance ratio $\Delta Z / Z$ of the as-prepared and field annealed $\mathrm{FeCoNi}$ electroplated wires.
Nevertheless, following the tradition of the GMI studies we shall use this term for the voltage/current ratio regardless of the degree of nonlinearity. The measuring system allowed us to make a Fourier analysis of the output voltage. We have measured the dependence of the Fourier components at the fundamental $F$ (frequency $f$ is equal to the exciting frequency $f_{\text {ex }}$ ), the first G1 $\left(f=2 f_{\text {ex }}\right)$ and second G2 $\left(f=3 f_{\text {ex }}\right)$ harmonics in the external magnetic field applied parallel to the wire axis.

Figure 1(a) shows the hysteresis loops of the samples. The annealing in the magnetic field reduces the saturation field but does not change the remanent magnetization. The magnetic field dependences of the output voltages in increasing field are shown in Fig. 1(b). The maximum MI ratios are very high: $\Delta Z / Z=820 \%$ for the as-prepared and $490 \%$ for the field annealed samples with the frequency of $1.5 \mathrm{MHz}$. There is a rather broad maximum at $H=-100 \mathrm{~A} / \mathrm{m}$ and a jump at $H=+100 \mathrm{~A} / \mathrm{m}$ for the field annealed sample. The hysteresis loop exhibits no visible peculiarity at $H=-100 \mathrm{~A} / \mathrm{m}$. The point is that the hysteresis loops are taken in longitudinal magnetic field but the change of the output signal is due to the response of the magnetic layer to the circumferential magnetic field induced by the ac current. The maximum values of the GMI ratio of the electroplated wires depend on the frequency of the driving current (Fig. 2). The as-prepared sample shows a very high $\Delta Z / Z$ ratio of about $820 \%$ at $1.5 \mathrm{MHz}$, which is higher than the $\Delta Z / Z$ ratio of the field annealed sample (maximum of about $500 \%$ ). The GMI ratio of the annealed wire increases up to $1 \mathrm{MHz}$ and almost does not depend on the frequency at the higher frequencies. If $f>5 \mathrm{MHz}$, the GMI of the field annealed sample is higher than that of the as-prepared sample. The weak dependence of the $\Delta Z / Z$ ratio of the field annealed wire in the wide range of frequencies may be important for applications.

The shape of the output signal is highly distorted especially in the external field near the GMI maximum (Fig. 3). This indicates the nonlinear behaviour and the generation of high harmonics in our $\mathrm{FeCoNi}$ electroplated wire and that the essential part in the output voltage is due to their appearance. To understand the origin of such a high GMI effect, we have made a Fourier analysis of the output voltage at a frequency of $1.5 \mathrm{MHz}$ where the highest GMI effect appears (Fig. 4). In the saturated state, the G1 and G2 amplitudes are much smaller than the $F 1$ amplitude for both samples. As the absolute value of the magnetic field decreases, the harmonics grow up to the GMI maximum (Fig. 5). We define the relative changes of the induced voltage as $\Delta U / U(H)=$ $100\left[U(H)-U\left(H_{\max }\right)\right] / U H_{\max }$. For each harmonic the relative change was calculated for $H_{\max }=1.5 \mathrm{kA} / \mathrm{m}$ (maximum field shown in Fig. 5). The relative changes in this field were: $450 \%$ for $F ; 600 \%$ for $G 1$; and about $30000 \%$ for $G 2$.

The first and second harmonics are noticeable only in the vicinity of the spin re-orientation transition points. It follows that the rather large $G 1$ and $G 2$ intensities result from the high sensitivity of the magnetic system to the circumferential magnetic field induced by the ac current near the transition points. To make sure that this is correct, we have calculated the high-frequency transverse susceptibility $\chi$ for the thin magnetic layer that covers the nonmagnetic wire. The details of the calculations will be published elsewhere. ${ }^{[17]}$ The magnetic field dependence of the imaginary part of the component of $\chi, \operatorname{Im} \chi-H$ curve, is very similar to the $U$ - $H$ curve. This confirms our interpretation. The simplest and direct evidence for the nonlinearity is the dependence of $Z$ on the electric current. Figure 5 shows the dependence of the GMI ratio on the driving current value. The maximum GMI ratio for the current of $10 \mathrm{~mA}$ is only $160 \%$ at the frequency of $1.5 \mathrm{MHz}$ in the as-prepared sample but the increase of the current intensity up to $35 \mathrm{~mA}$ results in an $820 \%$ GMI ratio at the same frequency. The origin of such a dramatic change is clear from Fig. 5 which shows the field dependence of the second harmonics for the different current intensities. The $G 2$ variation at the current of $10 \mathrm{~mA}$ is negligible 

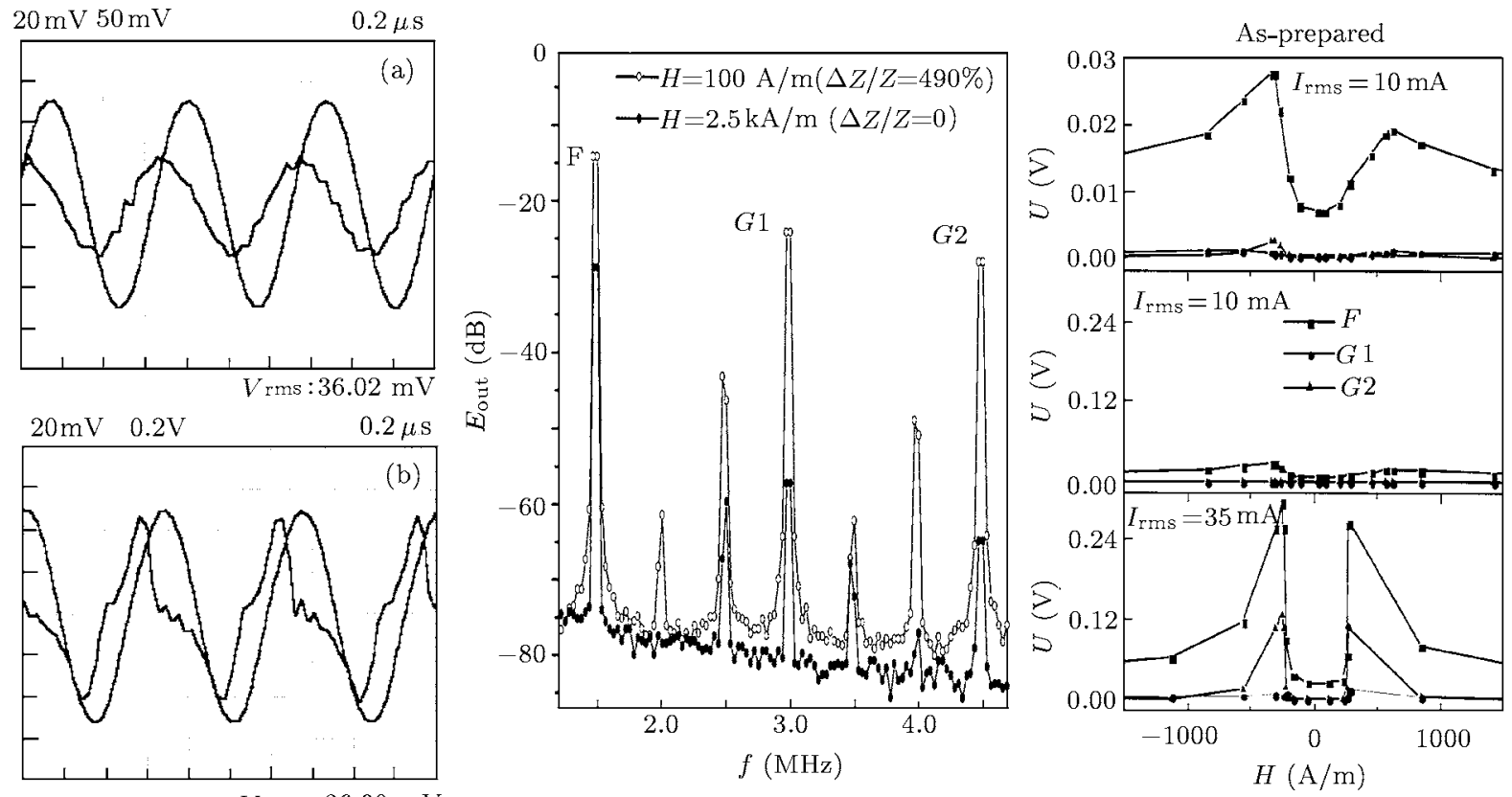

$V \mathrm{rms}: 36.00 \mathrm{mV}$

Fig. 3. Oscilloscope traces of the induced voltage for $6 \mathrm{~cm}$ FeCoNi electroplated wire without treatment; in the field of about (a) $-2.6 \mathrm{kA} / \mathrm{m}$ and (b) $-100 \mathrm{~A} / \mathrm{m}$ at a frequency of $1.5 \mathrm{MHz}$ [time axis is $1 / 6(\mu \mathrm{s} / \mathrm{div})$ ] and current of $I_{\mathrm{rms}}=35 \mathrm{~mA}$. The top is the current $I(t)$ trace.
Fig. 4. Fourier analysis of the voltage $U(t)$ induced across the FeCoNi electroplated field annealed wire in two different fields. The frequency of the driving current was $1.5 \mathrm{MHz}$ and the intensity of the ac current of $I_{\mathrm{rms}}=35 \mathrm{~mA}$. Three peaks at the fundamental $(F)$, first $(G 1)$ and second ( $G 2)$ frequencies are of interest to us.
Fig. 5. External field dependence of the Fourier components of the voltage of the $\mathrm{FeCoNi}$ electroplated wire for the currents of 10 and $35 \mathrm{~mA}$. The fundamental $F$ (frequency is equal to $f_{\text {ex }}=1.5 \mathrm{MHz}$ of the exciting signal), the first $(G 1)$ (at frequency $2 f_{\mathrm{ex}}=$ $3 \mathrm{MHz}$ ) and the second (G2) (at frequency $3 f_{\text {ex }}=4.5 \mathrm{MHz}$ ) harmonics are of interest to us. while the huge change in the second harmonic is seen at the current amplitude of $35 \mathrm{~mA}$. The voltage related to the second harmonic at $I_{\mathrm{rms}}=35 \mathrm{~mA}$ is four times greater than that related to the fundamental response at $I_{\mathrm{rms}}=10 \mathrm{~mA}$. The extraordinarily high (up to $820 \%$ of the magnetoimpedance ratio) and sensitive magnetoimpedance effect has been observed in FeCoNi magnetic tubes electroplated onto the $\mathrm{CuBe}$ nonmagnetic wire at frequency of about $1 \mathrm{MHz}$. The strong nonlinear effect has been found and studied. The high harmonics generation has been investigated. The first and especially the second harmonics show a much larger variation with the external magnetic field than the fundamental harmonics. The super high sensitivity of the harmonics is promising from an application viewpoint. The strong dependence of the GMI ratio on the intensity of the exiting current confirms the role of the nonlinearity in the formation of the very high GMI ratio.

\section{References}

[1] Landau L D and Lifshitz E M 1975 Electrodynamics of Continuous Media (Oxford: Pergamon) p 195

[2] Beach R S and Berkowitz A E 1994 Appl. Phys. Lett. 64 3652

[3] Panina L V and Mohri K 1994 Appl. Phys. Lett. 651189
[4] Sommer R L and Chien C L 1996 J. Appl. Phys. 795139

[5] Kurlyandskaya G V, Garcia-Beneytez J M, Vázquez M, Sinnecker J P, Lukshina V A and Potapov A P $1998 \mathrm{~J}$. Appl. Phys. 836581

[6] Kurlyandskaya G V, Vázquez M, Munoz J L, Garcia D and McCord J 1999 J. Magn. Magn. Mater. 197259

[7] Dai Y Y, Xiao S Q, Liu Y H, Zhang L, Wu H Z and Zhang Y Z 2001 Chin. Phys. Lett. 18272

[8] Chen D X, Munoz J L, Hernando A and Vázquez M 1998 Phys. Rev. B 5710699

[9] Usov N A, Antonov A S and Lagar'kov A N 1998 J. Magn. Magn. Mater. 185159

[10] Panina L V and Mohri K 2000 Sensors and Actuators A 8171

[11] Gromov A and Korenivski V 2000 J. Phys. D: Appl. Phys. 33773

[12] Beach R S, Smith N, Platt C L, Jeffers F and Berkowitz A E 1996 Appl. Phys. Lett. 682753

[13] Kurlyandskaya G V, Barandiarán J M, Muñoz J L, Gutierrez J, Garcia D, Vázquez M and Vas'kovskiy V O $2000 \mathrm{~J}$. Appl. Phys. 874822

[14] Garcia J M, Asenjo A, Sinnecker J P and Vazquez M 2000 J. Magn. Magn. Mater. 215-216 352

[15] Kurlyandskaya G V, Barandiarán J M, Gutierrez J, García D, Vázquez M and Vas'kovskiy V O, 1999 J. Appl. Phys. 855438

[16] Iida S, Ishii O and Kambe S 1998 J. Appl. Phys. 37 L869

[17] Kurlyandskaya G V, Yakabchuk H, Kisker E, Bebenin N, Garcia-Miquel $\mathrm{H}$, Vázquez $\mathrm{M}$ and Vas'kovskiy $\mathrm{V}$ O $J$. Appl. Phys. (submitted) 